\title{
CP violation and $D^{o}-\overline{D^{\circ}}$ mixing in charm photoproduction at Fermilab
}

\author{
S.P. Ratti * \\ University of Pavia and INFN, via Bassi 6, I-27100 Pavia, Italy \\ isergio.ratti@pv.infn.iti
}

Abstract: FOCUS is a high statistics photoproduction experiment at Fermilab. Among the many results that are being obtained in the context of the $\mathrm{CP}$ violation issue, we measured the values of the $D^{o} \overline{D^{o}}$ lifetime mixing parameter $y_{c p}(=\Delta \Gamma / 2 \Gamma)$ and compared the double Cabibbo suppressed amplitude $R_{W S}$ to the measurement of CLEO-II. We compared also particle antiparticle asymmetries in decay channels such as $D^{+} \rightarrow K^{-} K^{+} \pi^{+}$, $D^{0} \rightarrow K^{-} K^{+}, D^{0} \rightarrow \pi^{-} \pi^{+}$to other experiments.

\section{Introduction}

FOCUS is a high statistics photoproduction experiment at Fermilab. It collected over 7 billion triggers and fully reconstructed about 1.5 million charm decays; therefore it offers a very high sensitivity to investigate rare charm phenomena or eventually probe New Physics. For $D^{o}-\overline{D^{o}}$ mixing and $\mathrm{CP}$ violation in charm decays, the Standard Model predicts rates orders of magnitude below the sensitivities of current experiments [i]. Mixing occurs when the weak eigenstates $\left(D_{1}^{o}\right.$ and $\left.D_{2}^{o}\right)$ have different masses and/or differnt total widths. Direct

${ }^{*}$ Coauthors: J.M.Link, M.Reyes, P.M.Yager (UC DAVIS); J.Anjos, I.Bediaga, C.Gobel, J.Magnin, A. Massafferri, J.M. de Miranda, I.M.Pepe, A.C. dos Reis, (CPBF, Rio de Janeiro); S.Carrillo, E.Casimiro, A.Sánchez-Hernández, C.Uribe, F.Vasquez (CINVESTAV, México City); L.Cinquini, J.P.Cumalat, B.O'Reilly, J.E.Ramirez, E.W.Vaandering (CU Boulder); J.N.Butler, H.W.K.Cheung, I.Gaines, P.H.Garbincius, L.A.Garren, E.Gottschalk, P.H.Kasper, A.E.Kreymer, R.Kuschke (Fermilab); S.Bianco, F.L.Fabbri, S.Sarwar, A.Zallo (INFN Frascati); C.Cawlfield, D.Y.Kim, A.Rahimi, J.Wiss (UI Champaign); R.Gardner, A.Kryemadhi (IU Bloomington); Y.S.Chung, J.S.Kang, B.R.Ko, J.W.Kwak, K.B.Lee, H.Park (Korea University, Seoul); G.Alimonti, M.Boschini, B.Caccianiga, P.D'Angelo, M.DiCorato, P.Dini, M.Giammarchi, P.Inzani, F.Leveraro, S.Malvezzi, D.Menasce, M.Mezzadri, L.Milazzo, L.Moroni, D.Pedrini, C.Pontoglio, F.Prelz, M.Rovere, S.Sala (INFN and Milano); T.F.Davenport III (UNC Asheville); L.Agostino, V.Arena, G.Boca, G.Bonomi, G.Gianini, G.Liguori, M.M.Merlo, D.Pantea, C.Riccardi, I.Segoni, P.Vitulo (INFN and Pavia); H.Hernandez, A.M.Lopez, H.Mendez, L.Mendez, E.Montiel, D.Olaya, A.Paris, J.Quinones, C.Rivera, W.Xiong, Y.Zhang (Mayaguez, Puerto Rico); J.R.Wilson (USC Columbia); K.Cho, T.Handler, R.Mitchell (UT Knoxville); D.Engh, M.Hosack, W.E.Johns, M.Nehring, P.D.Sheldon, K.Stenson, M.Webster (Vanderbilt); M.Sheaff (Wisconsin, Madison) 
$\mathrm{CP}$ violation is investigated by looking at particle antiparticle asymmetries in several decay channels such as $D^{+} \rightarrow K^{-} K^{+} \pi^{+}, D^{0} \rightarrow K^{-} K^{+}, D^{0} \rightarrow \pi^{-} \pi^{+}$. In single Cabibbo suppressed $D$ decays, the penguin diagrams may provide the different phases of the two weak amplitudes and the direct $\mathrm{CP}$ violating asymmetries for these processes are expected to be at most $10^{-3}[\underline{2}]$.

The analysis presented here is mostly based on full data samples collected by the experiment during the Fermilab 1996-1997 fixed-target run with photons of $E_{a v} \approx 180$ $\mathrm{GeV}$ in a segmented $\mathrm{BeO}$ target, using an upgraded version of the E687 spectrometer [A] The vertex detector [16 layers of silicon microstrips: four $(\mathrm{x}, \mathrm{y})$ interleaved with the target segments and $12(\mathrm{x}, \mathrm{y}, \mathrm{u})$ downstream] provides excellent time resolutions $\sigma_{\tau} \approx 30$ fs. Charged particle momenta are measured from their deflections by two analysis magnets of opposite polarity and the hits left into five stations of multiwire proportional chambers. Three multicell threshold Cerenkov counters, two electromagnetic calorimeters, one hadron calorimeter and two arrays of muon counters provide particle identification.

\section{Mixing parameters}

The $\mathrm{CP}$ conserving mixing amplitude (squared) -i.e. for an initial $D^{\circ}$ - is written as:

$$
\left|<\overline{D^{o}}\right| D^{o}(t)>\left.\right|^{2} \approx e^{-\Gamma_{1} t}\left[1+e^{\Delta \Gamma t}-2 e^{\frac{\Delta \Gamma t}{2}} \cos (\Delta M t)\right]
$$

being: $\Gamma=\left(\Gamma_{1}+\Gamma_{2}\right) / 2$ the total decay width; $\Gamma_{1,2}$ the widths of the $C P= \pm 1$ states and $\Delta M$ their mass difference .

Defining $x=\frac{\Delta M}{\Gamma}$ and $y=\frac{\Delta \Gamma}{2 \Gamma}$, in the case of $|x|,|y|$ small, one can write the mixing amplitude as:

$$
A_{\text {mix }} \approx \frac{y+i x}{2} \Gamma t e^{-\frac{\Gamma t}{2}}
$$

Integrating $\left|A_{\text {mix }}\right|^{2}$ over time, the rate for the mixing process is described by:

$$
R_{\text {mix }}=\frac{\Gamma_{\text {mix }}}{\Gamma_{\text {unmix }}}=\frac{x^{2}+y^{2}}{2}
$$

Upper limits on $R_{\text {mix }}$ (e.g.: $95 \%$ confidence level) draw circles in the $x, y$ plane. Measurements of $R_{\text {mix }}$ from $\Delta M$ require accuracies in $\Delta M$ out of reach (less than $100 \mu \mathrm{eV}$ or so); measurements of $R_{\text {mix }}$ from $\Delta \Gamma$, require very good lifetime measurements. Measurements of $R_{m i x}$ are often performed by using semileptonic decays and looking at the wrong sign (hereafter WS) leptons. The particle-antiparticle ambiguity is solved by selecting the decays $D^{* \pm} \rightarrow D^{o} \pi^{ \pm}$, able to discriminate what $D^{o}$ is produced. Contrary to neutral kaons, hadronic charm decays are complicated by doubly Cabibbo suppressed (hereafter DCS) channels which add a term to the box diagram, as well as a new strong phase $\delta$ (relative to the Cabibbo favoured decay). The decay amplitude $R_{W S}$ is then written as:

$$
R_{W S}(t)=\left[R_{D C S}+y^{\prime} \Gamma t \sqrt{R_{D C S}}+\frac{x^{\prime 2}+y^{\prime 2}}{2} \frac{\Gamma^{2} t^{2}}{2}\right] e^{\Gamma t}
$$

where $x^{\prime}=x \cos \delta+y \sin \delta$ and $y^{\prime}=y \cos \delta-x \sin \delta$. When the mixing effects are very small, the branching ratio $R_{W S}$ is close to $R_{D C S}$ of the DCS decay. 


\section{Lifetime mixing}

The y parameter can be evaluated by measuring the lifetimes of pure CP states. Assuming $\mathrm{CP}$ conservation, $y_{c p}$, depends upon the width asymmetry $\Delta \Gamma$ between the $C P= \pm 1$ states. The discovery of two CP eigenstates, say $D_{1}^{o}$ and $D_{2}^{o}$ similar to the $K_{1}^{o}$ and $K_{2}^{o}$ states, is important "per se", independent of CP violation.

FOCUS measured $y_{c p}$ using the decay $D^{o} \rightarrow K^{-} K^{+}$as $C P=+1$ state and the CP mixed state $D^{o} \rightarrow K^{-} \pi^{+}$. Assuming $K^{-} \pi^{+}$as an equal mixture of $C P= \pm 1$ states, $y_{c p}$ can be written in terms of the two lifetimes:

$$
y_{c p}=\frac{\Gamma_{c p-\text { even }}-\Gamma_{c p-o d d}}{\Gamma_{c p-\text { even }}+\Gamma_{c p-o d d}}=\frac{\tau\left(D^{o} \rightarrow K \pi\right)}{\tau\left(D^{o} \rightarrow K K\right)}-1
$$
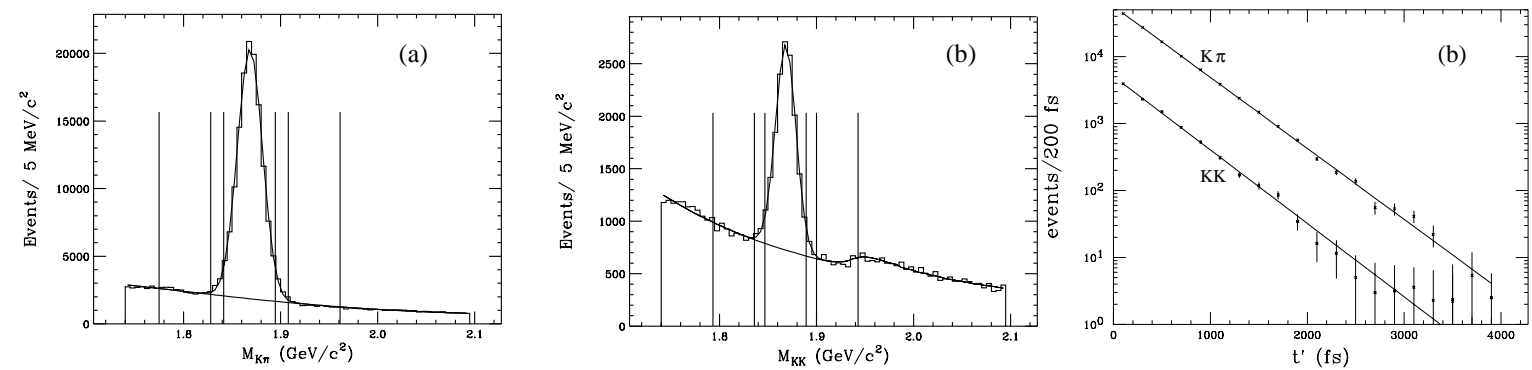

Figure 1: Mass and time distributions: a)- mass for $119738 D^{0} \rightarrow K^{-} \pi^{+}$events; b)- mass for $10331 D^{0} \rightarrow K^{-} K^{+}$events. Vertical lines show the selected signal and sideband regions for lifetime and $y_{c p}$ fit; c)- reduced proper time $t^{\prime}$ distributions for $D^{o} \rightarrow K^{-} K^{+}$(upper) and $D^{o} \rightarrow K^{-} \pi^{+}$ events. The distributions are background subtracted and include small Monte Carlo corrections.

To get $\tau\left(D^{o} \rightarrow K K\right)$ with an error of $\approx 1 \%$ one needs at least 10,000 events in the Cabibbo suppressed channel $D^{o} \rightarrow K^{+} K^{-}$. Fig. $\prod_{1}^{1}$ shows the mass distribuition for

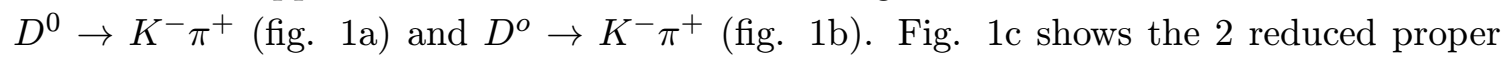
time distributions, with the binned-likelihood simultaneous fit using $y_{c p}$ and $\tau\left(K^{-} \pi^{+}\right)$as free parameters. The fit (20 bins, 200fs wide) spans over more than $6 \tau\left(K^{-} \pi^{+}\right)$. The

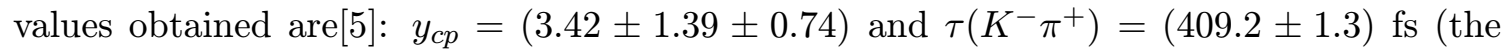
systematic error is nor relevant here). B. Yabslev[6] presented in this Session the BELLE value $) 0.5 \pm 1.0 \pm 1.0) \%$. The new average of $y_{c p}$ is definetly compatible with zero. It is important at this point the measurement of BaBar.

\section{Wrong sign and doubly Cabibbo suppressed decay}

Starting with over 200,000 $D^{*}$ events, FOCUS measured the WS branching ratio: $D^{o} \rightarrow$ $K^{+} \pi^{-}$or $\overline{D^{o}} \rightarrow K^{-} \pi^{+}$relative to their right sign (RS) decays. The sign of the $D^{*}$ identifies unambiguously the $\mathrm{C}$ state of the neutral meson but this choice reduced significantly the statistics. The event selection depends upon the capacity of separating pions from kaons. The relevant backgrounds are: $D^{o} \rightarrow \pi^{+} \pi^{-} ; D^{o} \rightarrow K^{+} K^{-}$; partially reconstructed D's and doubly misidentified $D^{o} \rightarrow K \pi$ decays. When the mass of the pion and the kaon are interchanged -leaving unchanged their charge- the final state produces a broad peak in the 
$\Delta M=M\left(D^{*}\right)-M\left(D^{o}\right)$ distribution at the charm mass. The $\Delta M$ vs $M(K \pi)$ scatter plot was divided into $80 \Delta M$ bins, each $1 \mathrm{MeV}$ wide, and the $K \pi$ mass distributions (RS and WR) were fitted in each bin the with the above backgrounds evaluated by MOntecarlo[i].]. The result is shown in fig. 2aja; from the fit we get $(149 \pm 31)$ WS events compared to $(36,760 \pm 195) \mathrm{RS}$ events. This provides a Branching Ratio:

$$
R_{W S}=\frac{\Gamma\left(D^{o} \rightarrow K^{+} \pi^{-}\right)}{\Gamma\left(D^{o} \rightarrow K^{-} \pi^{+}\right)}=(0.405 \pm 0.085 \pm 0.025) \%
$$
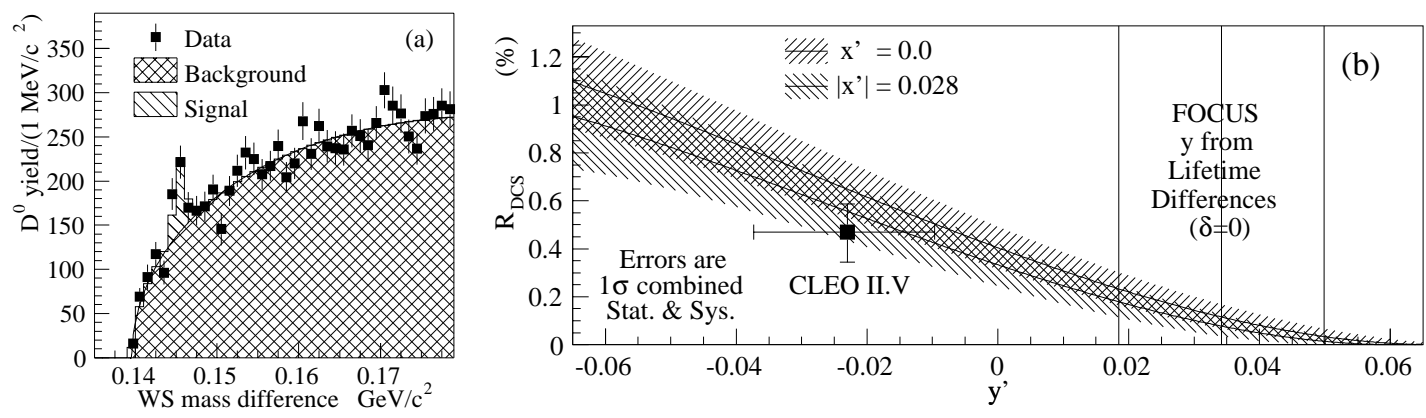

Figure 2: a) The $D^{0} \rightarrow K^{+} \pi^{-}$mass difference distribution with the signal and background contributions shown. b) $R_{D C S}$ plotted as a function of y'. Contours are given for two values of x' covering the $95 \% \mathrm{CL}$ of the CLEO result.

The time evolution for the $D^{o} \rightarrow K^{+} \pi^{-}$decay is given by equation $\left(\overline{2}-\overline{2}_{1} \mathbf{u}^{\prime}\right)$. If mixing is negligible, the branching ratio $R_{W S}$ provides a measurement of the DCS branching ratio, which is expected to be of order $\tan ^{4} \theta_{c} \approx 0.25 \%$. If mixing is significant, $R_{W S}$ depends upon the lifetime acceptance of our analysis. To investigate this point, in a Montecarlo sample of $D^{0} \rightarrow K^{-} \pi^{+}$decays, generated with a pure exponential and nominal lifetime, we re-weighted the accepted events by the ratio of the survival probability provided by its lifetime given by eq. (2.) and the probability of the pure exponential. We then obtained $R_{D C S}$ as a function of $x^{\prime}$ and $y^{\prime}$, which depend on our measurement for $R_{W S}$ and on the average values $\langle t\rangle$ and $\left\langle t^{2}\right\rangle$ obtained from our Montecarlo. We determined two bands in the $R_{D C S}-y^{\prime}$ plane shown in fig. 2 ing. They correspond to the two values of $x^{\prime}$ which cover the CLEO 95\% CL of $\left|x^{\prime}\right|<0.28$. For comparison, the CLEO ranges for $R_{D C S}$ and $y^{\prime}$ are also shown. The two experiments are compatible, although FOCUS may be suggestive of a possible $y_{c p}$ mixing different from zero.

\section{CP violation}

To investigate the $\mathrm{CP}$ violation issue, FOCUS selected the single Cabibbo suppressed decay modes: $D^{+} \rightarrow K^{-} K^{+} \pi^{+}, D^{o} \rightarrow K^{-} K^{+}, D^{o} \rightarrow \pi^{-} \pi^{+}$and their $\mathrm{C}$ conjugate states. The $D^{o}$ flavour is tagged by the $D^{* \pm}$ decays. These decays are normalized to their allowed modes to correct for the difference in the $D-\bar{D}$ photoproduction rates.

For instance, for the single Cabibbo suppressed decay mode $D^{0} \rightarrow K^{-} K^{+}$and the normalizing mode $D^{0} \rightarrow K^{-} \pi^{+}$the normalization is written as: $\eta(D)=\frac{N\left(D^{o} \rightarrow K^{-} K^{+}\right)}{N\left(D^{o} \rightarrow K^{-} \pi^{+}\right)}$. 
Here, $N\left(D^{o} \rightarrow K^{-} K^{+}\right)$is the efficiency corrected number of candidate decays. Assuming that there is no measurable CP violation in the Cabibbo favoured decays, the use of the $\eta$ ratio has the additional benefit that most of the corrections due to efficiencies cancel out, thus reducing the systematic uncertainties.

The CP asymmetries are then written as: $A_{c p}=\frac{\eta(D)-\eta(\bar{D})}{\eta(D)+\eta(\bar{D})}$.

Table 1: Measured CP asymmetries(10-2) (see Ref.

\begin{tabular}{|c|c|c|c|}
\hline Experiment & $\overline{D^{+}} \rightarrow K^{-} K^{+} \pi^{+}$ & $D^{o} \rightarrow K^{-} K^{+}$ & $D^{o} \rightarrow \pi^{-} \pi^{+}$ \\
\hline E687 & $-3.1 \pm 6.8$ & $+2.4 \pm 8.4$ & \\
\hline CLEO II & & $+8.0 \pm 6.1$ & \\
\hline E791 & $-1.4 \pm 2.9$ & $-1.0 \pm 4.9 \pm 1.2$ & $-4.9 \pm 7.8 \pm 3.0$ \\
\hline CLEO II["] & & $+0.05 \pm 2.18 \pm 0.84$ & $+1.95 \pm 3.22 \pm 0.84$ \\
\hline FOCUS [9.9] & $+0.6 \pm 1.1 \pm 0.5$ & $-0.1 \pm 2.2 \pm 1.5$ & $+4.8 \pm 3.9 \pm 2.5$ \\
\hline
\end{tabular}

In Table $I_{1}^{\overline{1}}$ the FOCUS results are compared to those from other experiments. All values are still consistent with zero but provide a substantial improvement over previous limits. No evidence of $\mathrm{CP}$ violation has been observed; new measurements on charm mixing will from FOCUS and CLEO as well as B-factories experiments such as BaBar and BELLE

\section{References}

[1] Compilation of Standard Model predictions: H.N.Nelson, hep-ex/9908021;

[2] a- I.I.Bigi and A.I.Sanda, "CP violation", Cambridge Univ. Press, UK, 2000) p. 382; bF.Buccella et al., Phys. Rev. D51, 3478 (1995);

[3] C.Caso et al., Eur. Phys. Jour. 15, 543-578 (2000);

[4] P.L.Frabetti et al., Nucl. Instr. Meth. A320, 519 (1992);

[5] J.Link, et al., Phys. Lett. B485, 62 (2000);

[6] B. Yabslev, paper n. 709 presented to this Conference;

[7] J.Link, et al., Phys. Rev. Lett. 86, 2955 (2001);

[8] A.B.Smith for CLEO coll., hep-ex 0104008, (2001);

[9] J.Link et al., Phys. Lett. B491, 232 (2000);

[10] a- P.L.Frabetti et al., Phys. Lett. B323, 459 (1994); b- ibidem B357, 678 (1995); c- Phys. Rev. Lett. 70, 1381; d- ibidem 1755, (1993); e- ibidem. 71, 827 (1993);

[11] S.P.Ratti et al. (FOCUS Coll.): a- Proc. Europ. Int. Conf. on H.E.P., HEP 99 (Ed.s K. Huitu, H. Kurti-Suonio, J. Maalampi, I.o.P, Bristol , 2001) p. 873; b- Proc. $30^{\text {th }}$ Int. Conf. on H.E.P. (Ed.s C.S. Lim, T. Yamanaka, World Sci., Singapore, 2001) p.381. 